\title{
Enterprise transformation projects in the financial industry and ethics (TPFI\&E)
}

\author{
Antoine Trad \\ Institute of Business and Information Systems Transformation Management, France
}

\section{Keywords}

Financial Activities, Ethics, Transformation and Artificial Intelligence, Machine Learning, Artificial Intelligence, Operational Research, Heuristics, Enterprise Architecture, Mathematical Model, Business Transformation Projects.

\begin{abstract}
The last three decades' global financial, societal and geopolitical crisis can be analysed by using an adapted holistic societal transformation framework. Sucha framework is able to deliver recurrent patterns of organized global financial misdeed models and their related crime schemes. The use of, an ethical approach, governance framework, enterprise architecture models and control services-based processes, are crucial to support such a complex crimes detection-based framework. The proposed framework uses measurable Critical Success Factors (CSF) and Critical Success Areas (CSA) which characterize the evaluation of risk factors related to Global Financial Predators Activities (GFPA), mainly misdeeds. This article presents the concept of a framework, that can be used for proactive detection and tracking of financial problems and misdeeds, which are organized and done by GFPAs. Such GFPAs are in general ranked as the most ethical organizations, because of such ranking organisations are chosen by major financial circles. In this article the author presents an artificial intelligence based decision-making concept that is the framework's kernel. This generic decision making concept uses a mathematical model, which manages various types of algorithms. A Transformation Project (or simply a Project), depends on, the capacities of the decision-making system, the profile of the Organizational/Societal Transformation Manager (or simply the Manager) and his team who should be supported by a holistic cross-functional framework. Project's complexity as well as the usage of underlying Decision-Making System (DMS) and enterprise architecture can be evaluated by a tuneable CSF based mathematical model. This framework's and related research works' originalities are that it can be used in any stage of the transformation project for the detecttion of any type of GFPA misdeed(s). The main limitation is the ongoing ethical and regulatory bodies that are governed by corrupt organizations and political bodies.
\end{abstract}

\section{Introduction}

Today finance related risks' management, ethical and legal standards are not mature and are even very chaotic. These facts (in the form of risk CSFs) can damage an organization's Project. The proposed framework is applied for financial risk management and is aimed mainly to support the proactive detection of financial irregularities, locked-in traps and major financial crimes, which can be fatal for an organization, enterprise and even a country (simply an Entity will be used). Some of major states and financial powers are responsible of most of GFPA crimes, and estonigshly, they even enjoy excellent world class reputations. In this article various cases are mentioned, because they are related to known financial centres, who enjoy top worldwide position in transparency and ethical rankings (Transparency, 2020; Swissinfo, 2021), and who in the same time have committed major financial irregularities and crimes. The main reason for this global contradiction is that they have overwhelming legal, political and financial advisory support, who block any attempt to divulgate such unethical behaviour. Therefore, questions arise, like: What are the roles of ethics, ranking, control, transparency and legal regulations? And are they credible? Are ethics and regulations bodies, in so-called ethical and advanced countries biased? Can Artificial Intelligence (AI) approach be used to dissimulate unethical and even illegal GFPA patterns of behaviour? 


\section{An AI Approach \\ Artificial Intelligence Basics}

$\mathrm{AI}$ is a concept that is much older than Information and Communication Systems (ICS) and its role is to inspect if it is possible to create computational units that contain human-like cognitive abilities, which would support Projects. It emerged as a practical domain in the middle of the $20^{\text {th }}$ century. In 1950, Alan Turing developed a fundamental test for computational intelligence, which is known as the Turing Test. The term AI was coined in the proposal for a seminal AI conference that took place at Dartmouth in 1956 (Schmelzer, 2021). AI's main domain is concentrated on learning processes, which can be used to support the TPFI\&E. The author's framework includes the Heuristic Decision Trees (HDT) that supports an incremental learning process.

\section{A Learning Process \\ The Path to Hyperautomation}

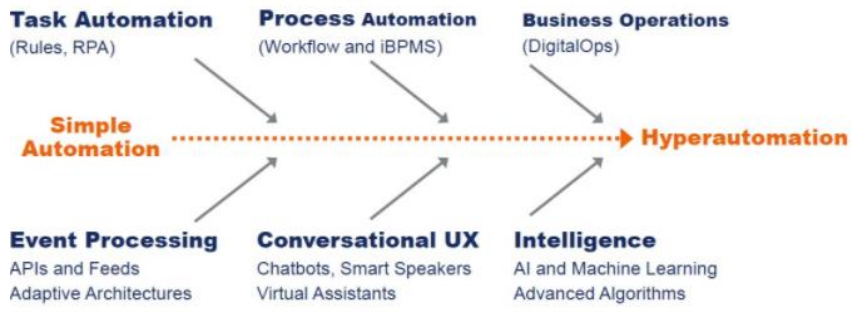

Figure 1. The growing role of OR on Hyperautomation (Kapoor, 2021).

The author's Transformation, Research and Architecture Development framework (TRADf) promotes techniques to ensure Project's success, by using: 1) Enterprise Architecture (EA) modelling; 2) Implementing Machine Learning (ML) with Operations Research (OR) components; 3) DMS' support; 4) Using a Generic AI Module (GAIM) as an interface for OR and ML operations; 5) Applying complex AI algorithmics; and 6) TPFI\&E to capture GFPAs' misdeeds.

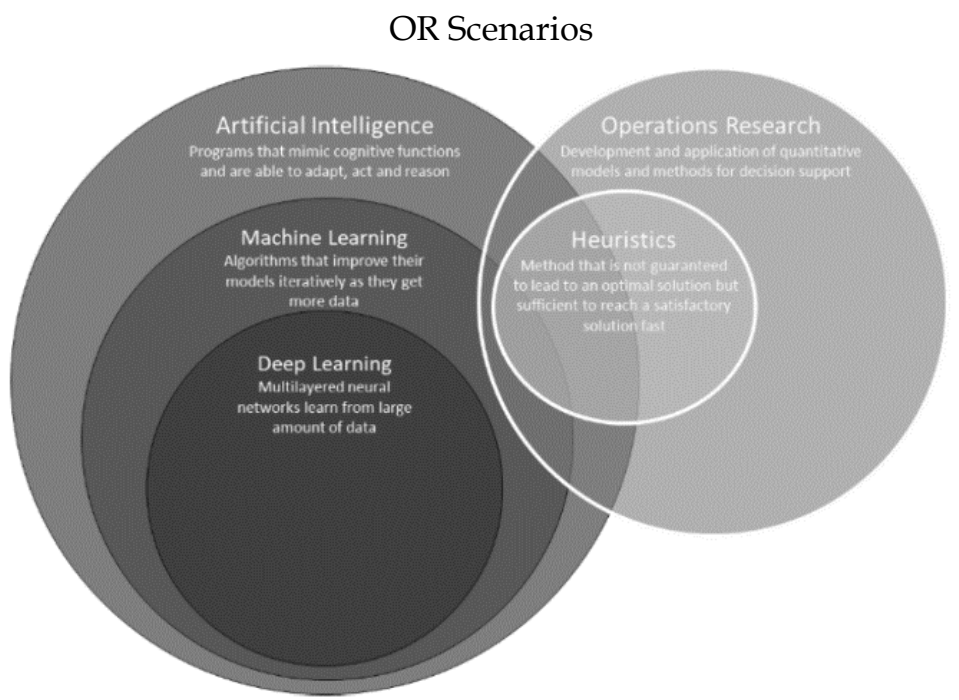

Figure 2. Subfields of AI and OR (Dornemann, Rückert, Fischer, \& Taraz, 2020)

The implementation of such Projects, requires significant skills in EA, forensics, financial auditing, DMS, AI and OR domains; and above all courage. GAIM handles OR calls and offers: 1) A generic algorithm architecture; 2) A generic services interfaces; and 3) Software modelling patterns. GAIM is a part of the TRAD $f$ and supports the Project resources to interact with all the Entity's EA phases, by using services oriented architecture. OR's main advantages and trends for 2021 are tremendous, as shown in Figure 1 (Kapoor, 2021); and is a leading domain in quantitative finance. AI emulates intelligent behaviour by using the tuneable Applied Holistic Mathematical Model for the Detection of GFPAs (AHMM4DGFPA). OR uses GAIM as an interface to interact with the Entity's EA models by using a Natural Programming Language 
(NLP) for the development of AI/OR scenarios. As shown in Figure 2, ML and OR scenarios are based on techniques similar to the HDT, that uses Artificial Neural Networks (ANN). ANN promotes iterative learning, which can be: 1) Supervised; 2) Semi-supervised; or 3) Unsupervised. OR methods are applied to deliver information for developing optimal decisions, for production or scheduling. Logistics uses OR for optimizing their processes, like for example, GFPA's detection and other types of financial crimes. OR is used to coordinate operations, processes and activities in Entities and is based on mixed methods combining quantitative models, with the aim to deliver the optimal solution for a planning problem. The main OR domains: Linear (LP), Mixed (MIP), and Non-Linear Programming (NP). It includes also simulations, Markov chains and game theory. OR uses two types of procedures: 1) Searching for the optimal solution by using algorithms, like, Simplex or the Branch and Bound method. These methods need heavy computing resources; 2) In case of heuristics, ML is based on HDT that offers a set of decisions and their possible consequences; where the HDT consists of nodes and branches. HDT's processing is based on GFPA's types of problems and predefined constraints. ML/OR is used to learn from various GFPA problems by recursively partitioning the source problem type into subsets, which constitutes a set of nodes. This approach is efficient for learning about GFPA's behaviour and for building corrective actions; where deep learning is a specialized form of learning.

\section{Deep Learning}

A subdomain of ML is Deep Learning (DL) that is also based on ANN. Where, DL focuses on implementing vast parametric approximators using Deep Neural Networks (DNN), which in turn uses a high number of layers to model complex, non-linear relationships between variables or CSFs. DL uses large datasets for predictive analytics in OR. Figure 2, illustrates AI's domains and their relation to OR, which need a GAIM and HDT to offer a generic learning approach. AI includes ML, OR, DL, intelligent algorithms and HDTs, like, genetic algorithms. AI and ML are used to complement OR's optimization functions, where $\mathrm{ML}$ and OR use iterative methods and are formulated as a constrained maximization or minimization process. In OR, an Objective Function (OF) defines the quality of TPFI\&E solution. A learning process is the optimization of a function, which is the measure of discrepancy between the output (meaning the predictions of the model being trained) and the solution's path and the target, which can be modelled using the HDT. A found solutions for GFPAs can be tuned using quantitative methods like Data Science (DS).

\section{Data Science and OR Basics}

DS represents the entire process of finding meaning in data by using ML and OR algorithms, which assists the HDT process, who is capable of tuning its learning process by using data sources, like financial crimes records. Europeans define OR, as Operational Research, whereas in the USA it is defined as Operations Research, in both case they carry the OR abbreviation. The terms Management Science (MS) and OR are combined as ORMS. The main OR characteristics are: 1) It was invented during World War II and is flourishing today; 2) The main domains are: optimization, production management, rescheduling, inventory management, and many other...3) Operations are activities of an Entity; 4) Research, is the process of analysis and testing supported by a scientific method; and 5) It is a quantitative approach to support the DMS in detecting GFPAs. OR supports the DMS by: 1) Using mathematical modelling, like the proposed AHMM4DGFPA; 2) Developing solution techniques; 3) To communicate results; 4) Provides rational bases for DMS in order to analyse complex TPFI\&E situations; and 5) It is an analytical and numerical tool. OR models can be used for TPFI\&E planning, inventory management, transportation, queuing systems and other.

\section{Major OR Models \\ Deterministic Models}

Deterministic models include: 1) NP, which is an optimization problem using an OF, which is nonlinear and/or uses a feasible region that is determined by nonlinear constraints; 2) LP can be applied to find optimal solution(s) and uses a linear $\mathrm{OF}$, which is conform to linear equality and linear inequality constraints. It's OF is a real-valued tuned function defined on an area; 3) In some situations, LP is not optimal like in discrete choices. For such situations, it is recommended to use Integer Programming (IP); 4) MIP is used for problems that include both discrete and continuous choices; 5) Network Optimization (NO) and IP are used for a large set of decision problems; and 6) Inventory Models make a decision that optimizes inventory cost and tries to reduce the total costs. 


\section{Stochastic Models}

Stochastic models include: 1) Markov process, which tries to proactively estimate changes in a defined period frame and their influence on the Project's behaviour, which has different phases and states. The probability of transition from one state to another is known and depends on the actual state and is independent of how the HDT reached a particular state; 2) Discrete-Time Markov Chains (DTMC) uses sets of just in time independent variables (CSFs), it is named the stochastic process, where CSFs' contents are influenced by the contents of actual depend variables and its past states. The process is continuous and is indexed by sets of natural numbers. The stochastic process uses a defined matrix, that contains the probabilities related to exchanging states. The used matrix includes the probability of having a targeted state, and actions to instantiate a new state. Most of stochastic processes that comply to Markov's constraints, that mainly demand that previous actions do not alter the process, but they can later the actual states' contents: 3) A Continuous-Time Markov chain (CTMC), is always an available stochastic process for all existing states, and it can modify their contents. The modification process is coordinated by an exponential random variable and afterwards it selects another state according to the behaviour stochastic matrix's probabilities; 4) Queueing Theory (QT) is a mathematical model that describes waiting objects, known as queues. A queue is created so that its maximum length and elapsed waiting time periods can be calculated. A QT is mainly an OR sub-domain, because its results are always processed to facilitate the decision-making process that supports the Project's activities and resources that are essential to support a defined business service; and 5) Many other application domains, like Data based Decision Analysis, Games Theory, Inventory processes, models and Simulation. The DMS uses OR models which can be deterministic or scholastic models.

\section{Deterministic Approach versus Stochastic Models}

Deterministic approach-based models, are based on the concept that Project's data are well known and will involve well-focused optimization activities, like stochastic processes which: 1) Use an explicit approach to represent unverified data which are represented in random variables (or CSFs); and 2) Use CSFs to evaluate system various types of risks and performances; to support models' development.

\section{Models Development}

Models in general and more specifically transformational models are representations of the real world and they are based on: 1) Three forms of models, the iconic, analogue and mathematical; 2) Iconic models are physical replicas of real objects; 3) Analogue models are physical in form; and 3) Mathematical models, like this chapter's AHMM4DGFPA, represent real world problems that are modelled by using a system of mathematical formulas, as shown in Figure 3, which a skeleton of a composite AI model.

\section{A Composite AI Model}

AI, DS, ML and OR are based on composite models' mechanisms, which are (Schmelzer, 2021): 1) The use of classifications that are in constant the evolution of items relationships; 2) OR which is optimal when combining it with DS and other AI domains; 3) TRADf goes further to combine them with EA, business engineering and decision making; and this Research and Development Project (RDP) proves this approach.

\section{The Research and Development Project RDP}

This RDP applies a cross-domain Literature Research and Evaluation Process (LR\&EP). The LR\&EP uses the author's specific qualitative analysis methodology to support this article's Experiment in the form of a Proof of Concept (E\&PoC). A CSA contains an enumerated set of CSFs. Each CSF contains an enumerated set of Key Performance Indicators (KPI) and a each KPI corresponds to a unique Project feature or requirement, where a requirement corresponds to a GFPA problem type to be solved. Each Project requirement or problem type has a default set of CSAs and corresponding CSFs which are managed by an OR analyst, who configures GAIM to assist the DMS. The AHMM4DGFPA based DMS applies processes to evaluate the real values of the CSA sets and deliver a possible set of solutions for GFPA problems and/or misdeeds.

\section{The AHMM4DGFPA}

The AHMM4DGFPA has a customizable and dynamic nomenclature to assist GAIM's integration by using also the Project's EA interface model. EA's main sub-system, the Architecture Development Method 
(ADM) supports the Project's development phases. The AHMM4DGFPA defines the Entity's overall structural model that supports a pool of parallelly synchronized OR threads' activities, in order to offer solutions that are possible to implement in a Project, and it also modifies the central matrix of CSAs, CSFs and KPIs. AHMM4DGFPA's nomenclature, which is shown in Figure 4 in a primitive form so that it can be understood. The DMS launches AHMM4DGFPA runtime instances which are responsible for solving Project's problems. This article uses a generic approach to OR that has a specific adaptation of the AHMM4DGFPA, which supports various types of transformation projects and their DMS processes. The AHMM4DGFPA uses a tree-based heuristics structure, aimed to support the evolution of an enterprise learning process. The AHMM4DGFPA based OR is used for the development of the enterprise information systems and their kernel decision-making system, which can be based on AI, OR and Machine Learning. The authors try to prove that a heuristics or action research's approach can generalize OR and other AI domains usage, by using generic HDT. TRAD $f$ is based on the AHMM4DGFPAs which is shown in Figure 3 and its underlying CSF management structure. The experiment uses the HDT and action research to solve GFPA problems related to an insurance case, which is used in the E\&PoC.

Basic Mathematical Model's (BMM) Nomenclature

Iteration
microRequirement
CSF
Requirement
CSA
microKnowledgeArtefact
neuron
microArtefact / neural network
microArtefactScenario
AI/Decision Making
microEntity
Entity or Enterprise
EnityIntelligence
BMM(Iteration) as an instance

$\mathrm{BMM}($ Iteration $)$ as an instance

\begin{tabular}{|c|c|}
\hline $\begin{array}{l}=\mathrm{KPI} \\
=\Sigma \mathrm{KPI}\end{array}$ & $\begin{array}{l}\text { (B1) } \\
\text { (B2) }\end{array}$ \\
\hline $\begin{array}{l}=\mathrm{CSF}=\underline{\mathrm{U}} \text { microRequirement } \\
=\Sigma \mathrm{CSF}\end{array}$ & $\begin{array}{l}\text { (B3) } \\
\text { (B4) }\end{array}$ \\
\hline $\begin{array}{l}=\underline{\mathrm{U}} \text { knowledgeItem }(\mathrm{s}) \\
=\text { action- }>\text { data }+ \text { microKnowledgeArtefact } \\
=\underline{\mathrm{U}} \text { neurons } \\
=\underline{\mathrm{U}} \text { microartefact } \\
=\underline{\mathrm{U}} \text { microArtefactScenario }\end{array}$ & $\begin{array}{l}\text { (B4) } \\
\text { (B5) } \\
\text { (B6) } \\
\text { (B9) } \\
\text { (B10) }\end{array}$ \\
\hline $\begin{array}{l}=\underline{\underline{U}} \text { microArtefact } \\
=\underline{\underline{U}} \text { microEntity }\end{array}$ & $\begin{array}{l}\text { (B7) } \\
\text { (B8) }\end{array}$ \\
\hline$=\underline{\mathrm{U}} \mathrm{AI} /$ Decision Making & (B11) \\
\hline$=$ EnityIntelligence(Iteration) & (B12) \\
\hline
\end{tabular}

The Generic AHMM's Formulation

AHMM

$$
=\underline{\mathrm{U}} \mathrm{ADMs}+\mathrm{BMMs}
$$

Figure 3. The applied AHMM4DGFPA's basics nomenclature (Trad \& Kalpić, 2020).

\section{Heuristics, Empirics and Action Research}

In this Project OR modules run on a pool of synchronized AHMM4DGFPA threads, in which, each AHMM4DGFPA thread launch's an HDT process (Della Croce, \& Camarinha-Matos T'kindt, 2002). Evaluations based on weightings and ratings mechanisms, supports the AHMM4DGFPA to process DMS' requests to find optimal solutions for a given GFPA problem. The AHMM4DGFPA is dispatcher for the OR's modules processing for problem solving and the synchronization of the set HDT instances, which are based on the following facts: 1) ANNs that are basically computing units based on the biological neural networks concept, constitute the central brain, which in turn uses sets of connected intelligent ANN nodes. ANNs try to simulate biological brain's neurons. The synapses or an ANN connection of a biological brain, transfers a signal to connected neurons; 2) OR algorithms can be considered as ML subset. These algorithms use various layers to synthesize higher-level requirements that are inputted, like in the cases of: 1) Image synthesisation. OR algorithms are supported by ANNs that include an iterative learning process. Learning processes are: supervised, semi-supervised or unsupervised. DL architectures use: DNN infrastructure, deep belief networks concepts, and others...; and 3) A learning process is an adaptation of the ANN, in 
order to optimize tasks responsible for pattern observations. The learning process includes the tunning of weightings of ANNs, in order to improve result's precision. A learning process is considered finished, when analysing supplementary observations does not decrease error's CSF significantly. This HDT approach is supported by TRADf.

\section{TRADf's Main Components and Characteristics}

The author's, TRAD $f$ can be used to evaluate risk and control of GFPA misdeeds; which uses CSA (and their corresponding CSFs) and the KPI must be selected and weighted to evaluate possible GFPA pitfalls and problems' risk, using an adapted mathematical model. The Research Question (RQ) is: "What is the impact of the GFPA on Entities and can we detect them?". (Trad, Nakitende, \& Afe Babalola, 2021). And: "What is the impact of the OR on Projects, and can a generic HDT be its basic structure?".

\section{Predators and Ethics}

The Nobel prize winner, the British economist, Angus Deatoon, warns about the destructive predator's professional graduating business schools and to stop this type of brutalities. The leading school with such a perception is the Chicago school, the Swiss HEC and many others (Le Monde, 2019). Such profiles can be classified as major GFPA profiles. The probable motivation is extreme cupidity which is destroying Europe's industrial and engineering cpacities. Revelations of the Swiss Leaks affair, the Swiss HSBC condemned for tax avoidance shows the need for an evolution towards ethical banking and that future generations of students in finance, economics and management, must be aware of ethical values. In this article it is related to many concrete cases, like, the Swiss: 1) Union des Banques Suisse (UBS) (Stupples, Sazonov \& Woolley, 2019), in which 32 trillion US dollars were hidden; 2) A gigantic fraud organized by the Swiss Fidusuisse, which shows the state of mind of such a GFPA accountant approach (Cornevin, 2020); and 3) Global tax fraud by Credit Suisse (Financial Times, 2021). As already mentioned, the AI based DMS approach, enables the localization of GFPAs.

\section{A Decision-Making Approach \\ The Role and Risks of Intermediaries}

Entities have standardized their audit, governance, control and monitoring environments. This proves that a TRADf is crucial for the Entities' financial subsystems for tracing GFPA acts. GFPA's Detection (GFPAD) combines AI, management sciences, audit, law and economics with Finance for Technologies (FinTech) services. GFPA depends on the structural, behavioural and cultural predispositions.

\section{Structural, Behavioral and Cultural Predisposition}

- The major problems with GFPAD are the following:

- National legal systems, used to ignore any attempt to investigate financial criminal acts.

- The Swiss Federal Court leaders of the far right-wing Swiss People's Party (SVP) that is Switzerland's ruling and most popular party, guilty of racism and anti-Semitism, over propagating extreme racism, using racist symbols (The Local, 2017). Racism based on the color of skin as shown in Figure 4. Where the black sheep denotes a colored person.

- GFPA based states tend to become leaders in FinTech.

- National Police and information services are used to block any attempt to pursue financial criminal acts. They even will attack the parties looking for deposits, like in the Libyan case. On Figure 4, the black sheep depicts foreigners and colored people... 


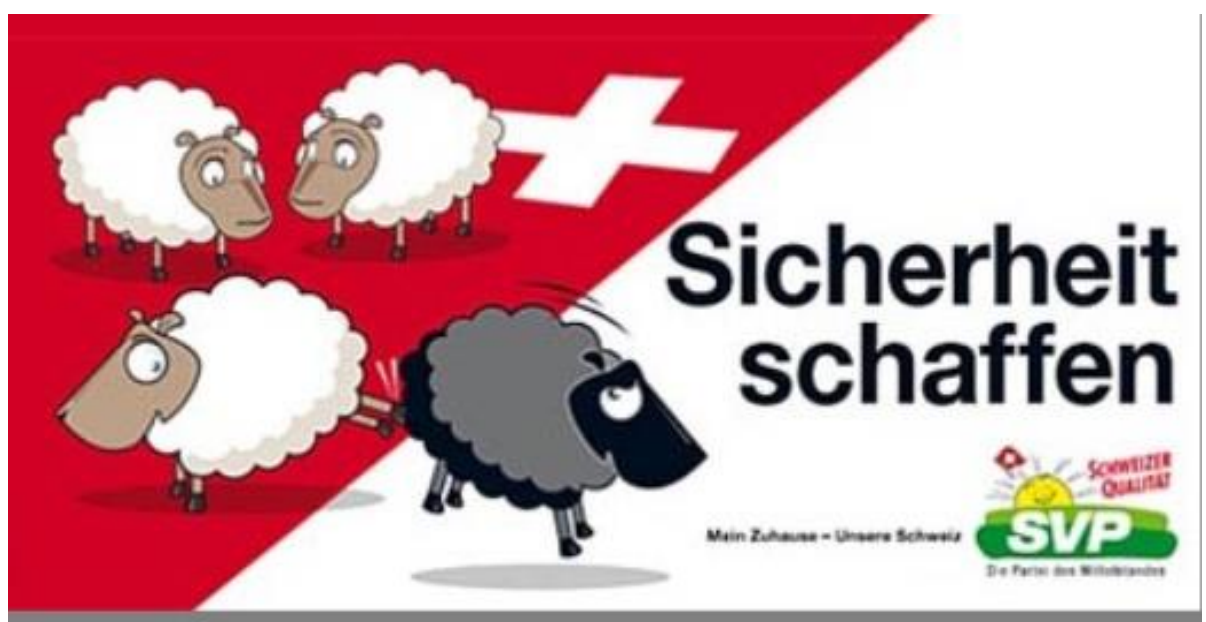

Figure 4. The Swiss ruling party's poster (The Local, 2017).

- Legal and Regulatory Constraints to Integrate: An adequate regulatory component must be integrated to support the GFPAD which can be integrated with the Entity's concept to combat fraud and financial crimes. The International Organization of Securities Commissions (IOSCO) identified eight domains that constitute FinTech. Before applying a FinTech strategy a Project or a transformation process must take into account ethics, financial crimes and irregularities.

- Organized financial crimes and irregularities.

Exponential Economic Growth of GFPA Entities

GFPA Entities organizes, abuses and plunders countries by destabilizing like the case of Lebanon, Malaysia, Mozambique and many other countries... This can be noticed by analysing Growth Domestic Product (GDP) diagrams. Analysing GDP diagrams' slopes and it seems obvious that the Lebanese declining GDP slope is inversely equivalent to the GFPA's. GFPAs are the unique beneficiaries from such tragedies.

\section{Classical GFPA Behaviour}

The classical GFPA behaviour is based on the following facts:

- The notion of states applying state crime exists, so various means are used to support GFPA actions.

- GFPA placed role in the Sri-Lanka civil war where GFPA crimes were done.

- Credit Suisse backs GFPA tactics in fraud worth $\$ 2$ billion in loans to Mozambique.

- In the case of Greece more 200 billion were plundered, while Greece is extremely suffering.

- Banks like the UBS is a GFPA and is due to the following facts: 1) It is the skeleton of the Swiss financial system; and 2) It hides 32 trillion US dollars and it is not declared (Stupples, Sazonov \& Woolley, 2019).

- GFPA's behavior is related to major fraud scandals, like the case of the UBS that was hit with a historic fine and this incredible Fraud crime (Alderman, 2019).

- GFPA accountancy crimes, like the ones committed by Swiss accountants are routine daily business (Cornevin, 2020).

- GFPA banks orchestrated the dilapidation of the victims of the Second World War.

- Banks' Influence. 


\section{Credit Suisse Group AG}

9,19 CHF

$-13,28(-59,11 \%) \downarrow$ od prvog dana

1. lis 17:30 CEST - Odricanje od odgovornosti
SWX: CSGN

+ Prati

\begin{tabular}{l|l|l|l|l|l|l|l} 
1D & $5 \mathrm{D}$ & $1 \mathrm{M}$ & $6 \mathrm{M}$ & $\mathrm{GDD}$ & $1 \mathrm{G}$ & $5 \mathrm{G}$ & Maks. \\
\hline
\end{tabular}

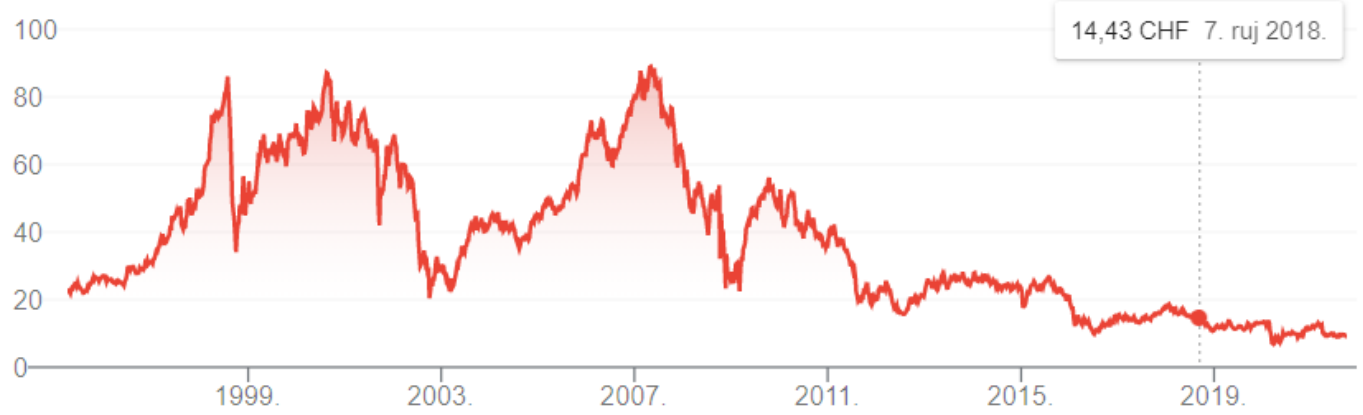

Figure 5. Shows Credit Suisse's downslide.

GFPA oriented banks' influence strategy is to:

- Destroy various banking and financial institutions worldwide, like the case of the Lebanese banking system.

- Prevent financial concurrent to get close to the immense Arab oil-based wealth.

Tax Fraud

- Sabotage of elite tourism that can endanger the Swiss one.

This section analyzes the notions of the GFPA's tax fraud mechanisms:

- There many GFPA Fraud cases that damage practically all countries, like the USA, France, Germany, Greece, and many other countries... The hidden capital is reused as a credit many countries.

- Corrupt transparency makes it impossible to mitigate risks.

- GFPA accountancy that blurs financial flows and to disable any type of transparency.

- Entities can easily slip in a locked-in situation and should try to avoid that.

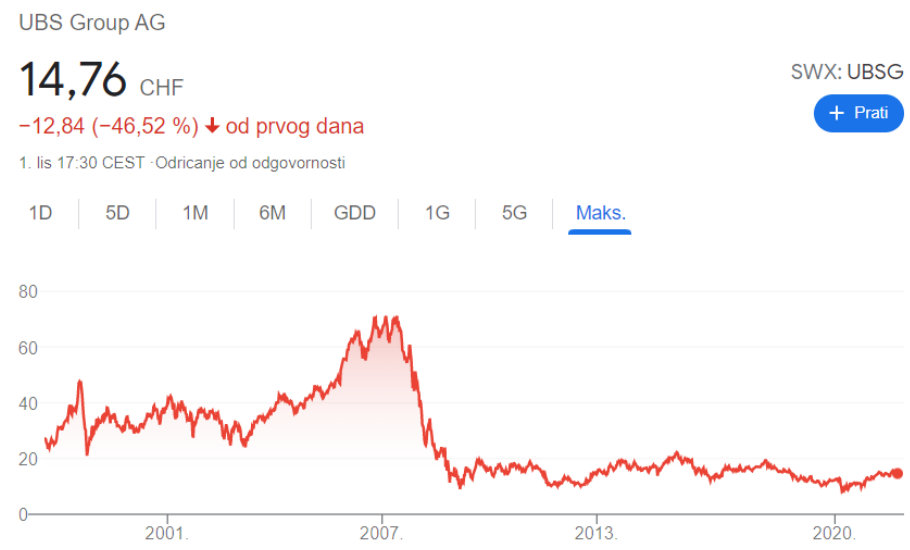

Figure 6. Shows UBS's downslide.

In fact, GFPAs are shortsighted and the cupidity resulted that related financial institutions were localized and have become a synonym for major financial crimes. Figures 5 and 6, clearly illustrated the collapse of these institutions' reputations and operational capacities. At the same time these financial 
institutions continue their GFPA behavior and in spite of the collapse of their values; they seem to have dissimulated illegal immense amounts of money (Stupples, Sazonov \& Woolley, 2019).

\section{Regulated Financial Systems}

Figures 5 and 6 show the cases of institutions that do not respect ethics and regulations; opposite to these two cases, the case of the French Société Général, shown in Figure 7, demonstrate clearly a wellcontrolled, human and ethical approach that is imposed by the French government and system. The French financial system can be considered as the most secure and reliable one; knowing that France is doing well in coordinating a global human, financial and industrial vision; such can become a raw model for the West and the world. These cases show clearly the need for an ethical approach, which is the main CSF for a longterm business and financial sustainable strategy. After applying an ethical financial system there is a need for a holistic security concept.

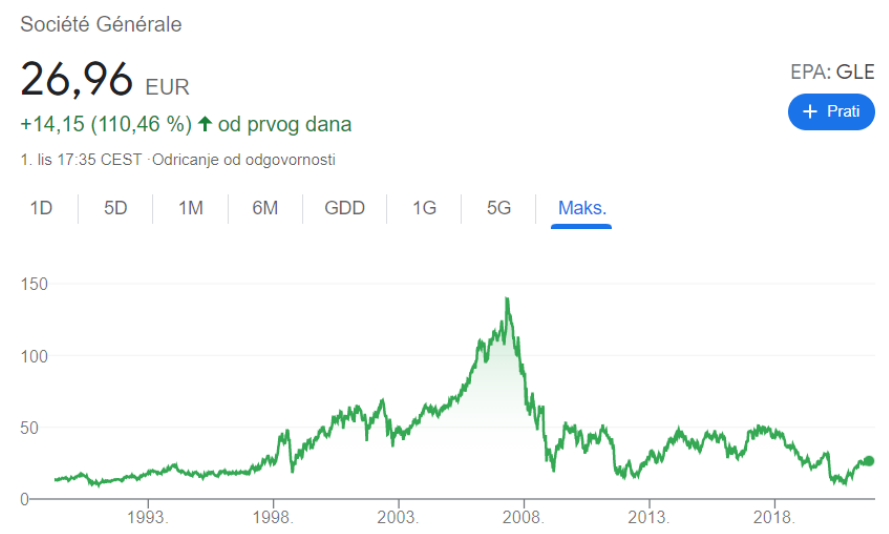

Figure 7. Shows the French Société Général robust approach.

\section{The Security Concept Cybersecurity Requirements}

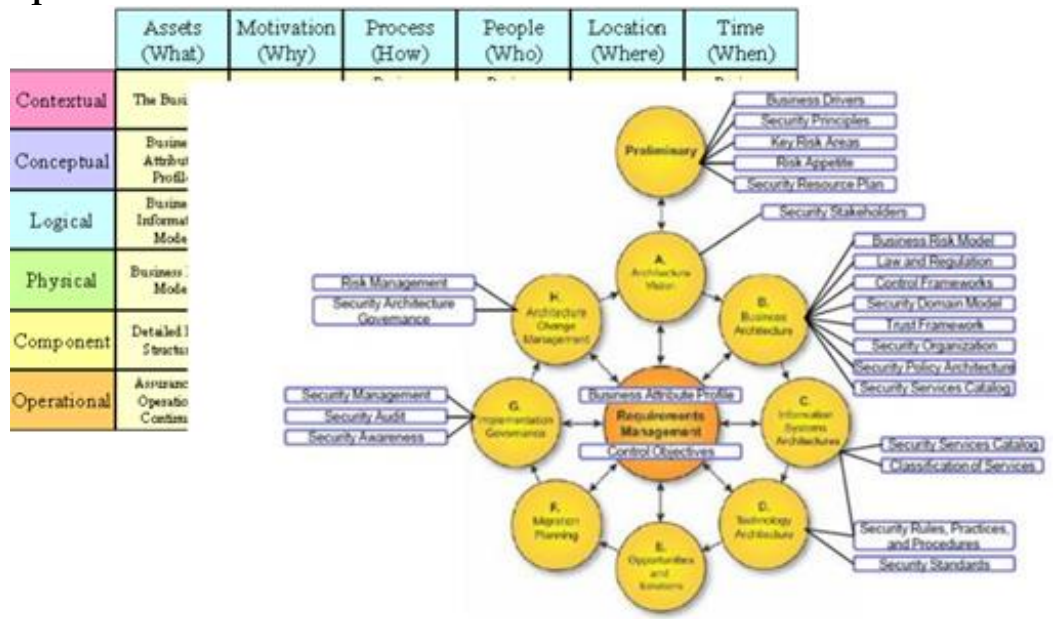

Figure 8. The architecture interface with security modules (Unwin, 2013)

Cybersecurity is the state of a business system that can be prone to any type of danger or threat; where the Project must deliver a system that is designed to provide maximum security (Oxford Dictionaries, 2017a). This section presents the CSFs that influence the TPFI\&E and this first CSA tries to argue the various aspects of cybersecurity requirements. Cybersecurity depends on the following fields: 1) Cyber technologies; 2) National security requirements; 3) International security requirements; 4) Organizational security requirements; and 5) Financial security and regulations. Cybersecurity requirements are the most fundamental for the Entity's business survival and they enclose various subdomains. The optimal cybersecurity architecture should fit in the company's global enterprise architecture framework that in turn is based on best practices. The resultant cybersecurity architecture is a mixture of technical solutions, business engineering, and security concepts. TOGAF includes sub-frameworks like the Sherwood Applied 
Business Security Architecture (SABSA) to handle cybersecurity requirements, as shown in Figure 8 (Unwin, 2013).

\section{Cybersecurity Domains}

Governance defines the interaction between various components and their Cyber or information technology security that understands the security of: 1) Data; 2) Technology resources; 3) Networks; 4) Web and Internet infrastructure; and 5) Applications, development and operations.

\section{Security Development and Operations' Integration}

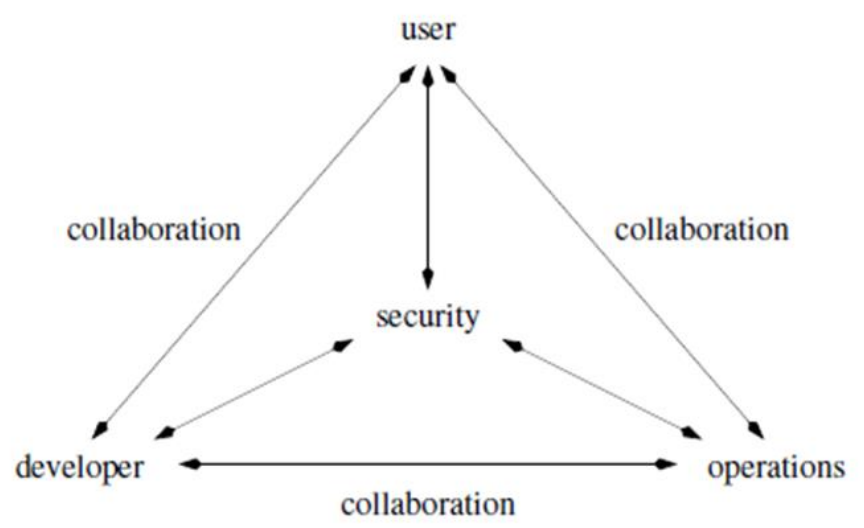

Figure 9. SecDevOps relationships between stakeholders (Mees, 2017)

Applications development and operations are coordinated by a secure development/operations (DevOps) process known as the SecDevOps. SecDevOps manages developers, operations and security Project members, as shown in Figure 9. The Project agile SecDevOps procedures to identify patterns for managing transformation requirements (Mees, 2017).

\section{Cybertransactions' Security Violations}

The European Commission defines a legislation to govern Cyberbusiness and progress has been done in its assertion. European Commission member states have implemented and enforced businessengineering related national practices. Cybertransactions outcomes have to be continually legally asserted, traced, and their periodic summaries are reported to the executive management (Fu \& Mittnight, 2015). Cyberbusinesses are orthogonal to cybersecurity requirements, where the business environment roles define the responsibility for enterprise's resources. Management of the enterprise's legal interests, resources, and accesses, should be managed by enterprise architect(s). Thus, the Cyberbusiness structure is an important consideration in the legal assertion and access management of Cybertransaction's security. The regulation for the Cybertransaction's security and law needs qualified time-stamps for robust (e)certification like those used in the European Union (European Union, 2014).

\section{Cybertransaction Law}

Cybertransaction is influenced by the Uniform Law Commissioners who promulgated the Uniform Electronic Transactions Act in 1999. It is the first adaptable effort to prepare a Cyberlaw for Cyberbusiness. Many countries have adopted Cyberbusiness regulations. The Uniform Electronic Transactions Act represents the first effort in providing some standardized rules and legislation to govern Cybertransactions. (The Uniform Law Commissioners, 2015).

\section{Cyberbusiness legislation assertion}

The integration of the business engineering module is done with the use of the standardized legal environment of The Open Group's Architecture Framework. This legal environment supports data protection laws, contract law, procurement law, fraud law and many other legislation domains to counter organized financial crimes.

\section{Financial Crime Model}

Brutal dictators like neo-Nazi brigands have a special status in states where the ownership of substantial financial assets can remain anonymous. Some Third World dictators maintain strong financial relationship to banks in financial havens. Some of these banks have even been established by criminals 
emerged from former wars. An example can be the notorious Nazi banker Francois Genoud (Brown, 2016). A country where the money cannot be transparently audited can provide security to dubious investors, although otherwise the same country may serve as role model of law obeying common citizens. Some of financial havens and their financial institutions have been the main leaders in worldwide financial scandals, misdeeds, and criminal acts including: 1) The Libor manipulation; 2) Currency manipulations; 3) credits manipulations; 4) Supporting arms dealing transactions; 5) Hijacking people's wealth; 6) Financial fraud; 7) The subprime crisis; 8) War victim wealth confiscation; 9) Tax evasion and Fraud; 10) Drug dealing financial support; 11) War support against future financial competitors; 12) Forced confiscations; 13) Drastic fines; and 14) Arms dealing; the major problem with combating such a system is that some Entities have hermetically closed system characterised by the following attitudes:

- Police and information services, blocking any attempt to pursue financial criminal acts.

- The legal system, ignoring any attempt to investigate financial criminal acts.

- Legal support too expensive, to discourage any action of law enforcing.

- Psychological harassment, to discredit investigators.

- Intolerance and discrimination, to block any foreign request.

- A powerful global network, to embed and hide various dubious operations.

- Financial guerrilla-like and hit and run tactics, to confiscate wealth.

- Occurrence of financial locked-in situations.

- Some financial haven states target to become leaders in FinTech, which is not very assuring; because FinTech should combat state criminality and enforce Cybersecurity international law.

\section{Cybersecurity International Law}

Facts show that international law on Cybersecurity is inefficient and is actually agonizing. Advanced Entities are hesitant to integrate international law that is based on the emergence of non-government normmaking initiatives. States insist on their traditional central legal system that marginalizes the inter-state governance of Cyberspace (Mačák, 2016). It is strongly recommended to avoid any form of financial collaboration with GFPA oriented organizations in countries acting as financial and business havens. France and the European Union are optimal models for innovation and global activities.

\section{The experiment as a proof of concept}

The Implementation Environment

The E\&PoC is implemented using the TRAD $f$ which uses AI based microartefacts on the basis of the " $1: 1$ " mapping concept

\section{CSFs, Rules and Constraints Setup}

OR's integration process execution starts with the use of the inputted data collection in the TRADf's data storage and then these data are filtered using the selected set of CSFs. The execution of the HDT follows. The inputted data collection is considered to be the root or initial node that helps in the establishment of the basic state that is enhanced with the adopted solution(s). The HDT reasoning goal is to select the optimal solution(s).

\section{RDP's Phases 1 and 2}

The Project's enumeration of CSAs were processed using TRADf. This E\&PoC uses Project's components, having a constraint, that the target CSA average must be higher than 7.5. The E\&PoC in Phase 2 , has given the average of 8,75, as shown in Table 1. The RDP and E\&PoC, are based on the AHMM4DGFPA that can adapt to any type of GFPA problem type by integrating a set of algorithms. This RDP's RQ is qualified by using a E\&PoC, that was implemented by employing Microsoft's VS2020, JEE and an EA tools. The E\&PoC contains the TRADf's major components for OR's processing that is based on a HDT mixed reasoning engine. The OF calculates the best solution for the encountered problems and a set of recommendations is presented. The deductions were done by using the analysis of all the selected CSAs, where a TRAD $f$ s NLP script is used, in which all its CSFs are stored and appear in Table's $11^{\text {st }}$ column. The TRADf's scripts in the background, are automated to calculate the weightings and ratings; known as the KPIs and a value from the enumerated sets; and they are tuned and stored in column 2 . This RDP's concept proposes a standardized and automated manner to evaluate LRPs, what is an evolution in regard to the very subjective method that is used in general, which may or may not make sense. If the automated LR\&EP's 
evaluation is successful, only then the experiment can be completed. The TRAD $f$ and its RDP automates complex RDP's feasibility in phase 1, and estimates the values for each selected KPI, as described in the authors' related works; on how to use CSA, CSF, KPI processing in the RDP. AHMM4DGFPA's main constraint is that CSAs for simple research components, having an average result below 8.5 will be ignored. In the case of the Machine Language's implementation an average result below 6.5 will be ignored. As shown in Table 1, the results justify (with an average of 8,75) the usage of the OR and HDT and how it can be used with the final E\&PoC or phase 2. AHMM4DGFPA's instances support the OR process, by using CSFs weightings and ratings (in phase 1), and are based on multicriteria evaluation (selected and defined constraints). Based on the LR\&EP and the related evaluation processes the most important CSFs are used and processed by the internal heuristic engine (Trad \& Kalpić, 2020). CSFs have bindings to specific RDP resources, where the AHMM4DGFPA formalism was designed to launch NLP microartefacts. The results in Table 1 show clearly that the OR proposal is feasible. The HDT represents the relationships between RDP's requirements, NLP generic and microartefacts, unique identifiers and the CSAs. The E\&PoC uses the $T R A D f$ client's interface. From the TRAD $f$ client's interface, the NLP development setup and editing interface can be launched. Once the development setup interface is activated, the NLP interface can be launched to implement the needed microartefact scripts to process the defined CSAs. These scripts make up the kernel DMS set of actions that are processed in the background.

\begin{tabular}{|c|c|c|c|}
\hline Critical Success Factors & KPIs & & Weightings \\
\hline CSF_TPFI\&E_GFPAD & Feasible & 7 & From 1 to 10.09 Selected \\
\hline CSF_TPFI\&E_AI_Approach & Mature & $=$ & From 1 to 10.09 Selected \\
\hline CSF_TPFI\&E_RDP & Complex & - & From 1 to 10.08 Selected \\
\hline CSF_TPFI\&E_TRADf & Proven & 7 & From 1 to 10.10 Selected \\
\hline CSF_TPFI\&E_Financial_Crimes & Feasible & - & From 1 to 10.09 Selected \\
\hline CSF_TPFI\&E_Security_Concept & Feasible & 7 & From 1 to 10.09 Selected \\
\hline CSF_TPFI\&E_EA_Concept & Complex & - & From 1 to 10.08 Selected \\
\hline CSF_TPFI\&E_Integration_PoC & Complex & - & From 1 to 10.08 Selected \\
\hline
\end{tabular}

Table 1. The sheet that concludes phase 1 with an average 8.75

\section{The Tree Processing and Resources and Possible Solutions}

OR's HDT contains a collection of nodes that uses the following artefacts and resources: 1) Actions; 2) Constraints; 3) Problem types to solve; and 4) A set of possible solutions and recommendations. A tree node contains is a CSF suggestion that is linked to a an actual HDT state. A state contains an aggregate of a resource linked with a 1:1 mapping link. The selected CSFs were fed in the HDT in order to reveal the optimal OR prerequisites for a selected problem. The CSFs were configured and weighted; afterwards they were processed in order to deliver a set of possible solutions. The HDT is launched with a default set of CSFs that map to a GFPA problem type. Then the HDT deliver a set of of possible solutions which are in fact transformation steps (Vella, Corne, Murphy, 2009). As shown in Figure 10, the E\&PoC's layers are: 1) EA using TOGAF and GAIM to link OR to various artefacts; 2) The client side includes modules which interface: Relational Databases, Various types of spreadsheets, Standard Files Interfaces and a first teer interface; 3) Data management services level contains a dispatchment hub; 4) The resources management level contains the following set of modules: A database entity-relational model, an XML based extensible mark-up language transformer; 5) The system level contains: monitoring component based on a generic log management system; and 6) The technical platform level contains the following modules: An portable and open Entity service bus, an object database interface, and a JEE data management module. This E\&PoC's HDT uses Google's OR library (Google Developers, 2021) and serves to confirm the research's RQ. The used OF finds the best solution for the selected problems. The E\&PoC's results are delivered as a set of solution and recommendations. OF helps to find the best solution to a problem; in this E\&PoC an Entity delivers packages to end-system's end-users. In a day, it assigns modules to financial inspections, afterwards is 
processes to deliver the optimal solution. The usage of modules and its stocked experience processed from the various cases of financial crimes and mapped to selected CSFs.

The E\&PoC's main difficulty is the selection of the assignments modules and similar cases having optimal solutions, like detected and judged cases. The LP algorithm has the following elements: 1) OF is related to the optimized quantity and is to minimize GFPAs; 2) The best solution is the one with the best value of the OF's (having maximum or minimum); 3) The defined constraints are the restrictions on solutions, based on the problem type, like if the Entity cannot assign modules superior to a defined weighting; and 4) A feasible solution is one that satisfies all the defined constraints for the problem type, without necessarily being best. The OF and the constraints are given by linear expressions, which makes this a linear problem and for the NLP script the steps for setting up and solving a problem are the following: 1) Import the required libraries; 2) Integrate the solver; 3 ) Define the variables, constraints and OF; 3) Invoke the OR solver and offer solutions. The next step is to identify the OF and constraints; using LP, in which the $\mathrm{OF}$ and the constraints are defined as linear expressions, like to maximize the OF goals to validate the set of constraints. As already mentioned, The LP algorithm is called from HDT's node, like all other quantitative methods. The LP algorithm receives an input array of arguments that contains: 1) The OF; 2) The set of constraints; and 3) Decision variables. The LP function is called from HDT's node to generate a set of new HDT nodes and offers the best solution in a given node.

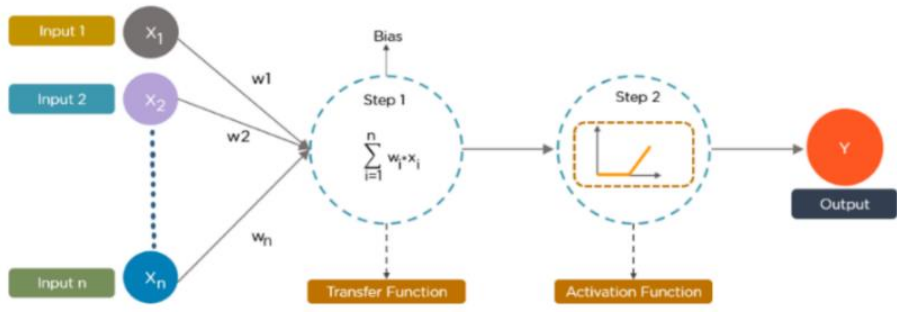

Figure 10. The activation of $\mathrm{OF}$.

\section{Conclusion}

The RDP is based on OR and presents the GAIM to interface other Project's resources. There are several trends shaping the future of OR; first, OR tasks in the life-cycle are automated that increases the Entity's stability and GFPAD. Another important element is that OR resources become accessible to more domains. GAIM has the ability to make OR algorithms transparent, that makes Project's oversight possible. The most important recommendations are: 1) Define OR's default CSFs and CSAs; 2) AI based Projects should be supported by OR tools that can integrate with the EA principles; 3) The integration of data standards is crucial, and OR must integrate various data standards; 4) GAIM enables OR's usage, that glues the various OR algorithms; 5) OR's integration needs a meta-model and a synchronized mapping concept; 6) Unbundling through the use of GAIM services in the context of a system that applies a holistic approach; 7) Use data management services to synchronize the implementation and integration of data models; 8) Use data transformation models to identify the major relationships between elements of the data models and data sources; 9) Design a generic data normalization and interoperability concept that uses the major market standards; 10) Prepare ADM's integration with the Project's data building and solution blocks concepts; 11) Define the overall OR constraints and rules; 12) Design the Project's conceptual views that are implemented to enable the usage of OR modules; 13) Generate common class diagrams for the OR(s) to be the building block that is designed in the initial phase and which is tuned in incoming phases; 14) Create in-house HDT algorithms that can replace commercial OR tools and support ML; and 15) OR and HDT can support activities related to transparency, fraud and corruption, especially the ones related to effective exchange of crucial data (OECD, 2011). In this article the focus is on the GFPAD, which proposes a concept to detect financial crimes and locked-in situations that from financial predators, like banks who made impressive but illegal trillions gains. The evolution of ethics in finance might bring an end to such financial manipulations and eventually bring to trial GFPA Entities for committing major crimes against humanity. These crimes caused the deaths of hundreds of thousands of people and the looting of their belongings. Some countries can be taken as an example, like France, which has convicted the Swiss UBS with a multi-billion euros fine for fraud and tax evasion; and is an example to followed. The GFPAD is used to transform a financial environment where Entities have been implementing control measures to respond to major risks, legal 
problems and challenges in relation with GFPA crimes. The GFPAD's E\&PoC showed that such a concept is feasible and offers the following set of recommendations:

- The Manager must deploy AI based GFPAD to enforce an anti-locked-in and detect crimes approach.

- The GFPAD team skills encompass the knowledge of: 1) Financial engineering and the needed logging, monitoring and assertion concepts; and 2) governance and controls integration to detect GFPA misdeeds.

- To design and implement a GFPAD subsystem, there is a need to implement a decision system.

- Global transparency organizations must be equitable and treat countries equally.

- Entities should avoid any form of financial collaboration with GFPA oriented organizations.

- France and the European Union have optimal models for innovation, education, ethical standards and global activities.

\section{References}

Alderman, L. (2019). French Court Fines UBS \$4.2 Billion for Helping Clients Evade Taxes. The New York Times. USA. Retrieved from https://www.nytimes.com/2019/02/20/business/ubs -france-tax-evasion.html

Brown, J. (2016). Wafic Said: businessman, philanthropist and political fixer. Financial Times. Retrieved on May 3, 2017, from: https://www.ft.com/content/a3cb764a-ecf1-11e5-bb79-2303682345c8

Cornevin, Ch. (2020). La police démantèle un vaste système de blanchiment de fraude fiscale... [Police dismantle massive tax fraud laundering scheme]. Le Figaro. France. Retrieved from https://www.lefigaro.fr/actualitefrance/la-police-demantele-un-vaste-systeme-de-blanchiment-de-fraude-fiscale-20200110

Della Croce F, T'kindt V (2002). A recovering beam search algorithm for the one-machine dynamic total completion time scheduling problem. J Oper Res Soc 2002.

Dornemann J, Rückert N, Fischer K, Taraz A. Artificial intelligence and operations research in maritime logistics. Econstor. Leibniz Information Centre for Economics. Germany, 2020

European Union (2014). Regulation (EU) No 910/2014 of the European Parliament and of the Council - on electronic identification and trust services for electronic transactions in the internal market and repealing Directive 1999/93/EC. The European Parliament and of the Council - Regulation. European Union.

Financial Times (2021). US Senate probes Credit Suisse after allegations of tax settlement breach. Financial TimesNikkei. USA.

Fu, Zh., \& Mittnight, E. (2015). Critical Success Factors for Continually Monitoring, Evaluating and Assessing Management of Enterprise IT. ISACA. Retrieved on May 9, 2017, from:

http://www.isaca.org/COBIT/focus/Pages/critical-success-factors-for-continually-monitoring-evaluating-andassessing-management-of-enterprise-it.aspx

Google Developers (2021). Get Started with OR-Tools for C\#. 2021. Google. https://developers.google.com/optimization/introduction/dotnet

Kapoor A. Artificial intelligence and machine learning: 5 trends to watch out for in 2021. AI Zone. 2021 DZone. https://dzone.com/articles/artificial-intelligence-amp-machine-learning-5-dev

Le Monde (2019). Le Prix Nobel d'économie Angus Deaton : Quand l'Etat produit une élite prédatrice [Nobel Lauréate in Economics Angus Deaton : "When the state produces a predatory elite]. Le Monde. Retrieved from https://www.lemonde.fr/idees/article/2019/12/27/angus-deaton-quand-l-etat-produit-une-elitepredatrice_6024205_3232.html

Mačák, K. (2016). Is the International Law of Cyber Security in Crisis? Law School-University of Exeter. Exeter, United Kingdom. Cyber Power. 8th International Conference on Cyber Conflict. Tallinn: Estonia: NATO CCD COE Publications.

Mees, W. (2017). Security by Design in an Enterprise Architecture Framework. Royal Military Academy, Department CISS. Renaissancelaan 30, 1000 Brussel. Belgium: NATO.

OECD (2011). Global forum on transparency and exchange of information for tax purposes peer review: switzerland 2011, Phase 1. OECD Publishing, Paris, France 2011.

Oxford Dictionaries (2017a). Security. London: Oxford Dictionaries. Retrieved on September 3, 2017, from: https://en.oxforddictionaries.com/definition/security

Schmelzer R. (2021). Data science vs. MLI vs. AI: How they work together. TechTarget 2021.

Stupples, B., Sazonov, A., \& Woolley, S. (2019, July 26). UBS Whistle-Blower Hunts Trillions Hidden in Treasure Isles. Bloomberg. Retrieved from https://www.bloomberg.com/news/articles/2019-07-26/ubs-whistle-blower-huntstrillions-hidden-in-treasure-islands

Swissinfo (2021). Switzerland diplomatically rejects Biden's 'fiscal paradise' label. 2021 Swissinfo. 
https:// www.swissinfo.ch/eng/business/diplomacy_switzerland-diplomatically-rejects-biden-s--fiscalparadise--label-/46578996

The Uniform Law Commissioners (2015). Electronic Transactions Act Summary. New York: The Uniform Law Commissioners. The Local (2017). SVP ad ruled racist by Swiss supreme court. The Local. https://www.thelocal.ch/20170413/svp-ad-ruled-racist-by-swiss-supreme-court

Trad, A., \& Kalpić, D. (2020) Using applied mathematical models for business transformation. IGI-Global. USA 2020.

Trad, A., \& Nakitende, M., \& Afe Babalola, T. (2021). Tech-based Enterprise Control and Audit for Financial Crimes: The Case of State Owned Global Financial Predators (SOGFP). IGI Global. USA.

Transparency (2020). CORRUPTION PERCEPTIONS INDEX. Transparency International. https://www.transparency.org/en/cpi/2020/index/nzl

Vella, A., Corne, D., \& Murphy, C. (2009). Hyper-heuristic decision tree induction. Sch. of MACS, Heriot-Watt Univ., Edinburgh, UK. Nature \& Biologically Inspired Computing, 2009. NaBIC 2009. World Congress.

Unwin, D. (2013). Security Architecture-Enterprise Architecture. Business Aspect. 